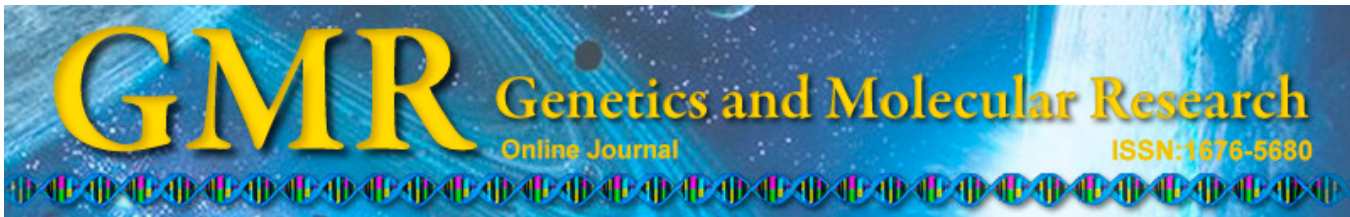

\title{
A novel $F B N 1$ heterozygous mutation identified in a Chinese family with autosomal dominant Marfan syndrome
}

\author{
Y. Yin ${ }^{1 *}$, X.-H. Liu ${ }^{2 *}$, X.-H. Li ${ }^{3}$, N. Fan ${ }^{2}$, D.-F. Lei ${ }^{4}$, Y. Wang ${ }^{2}$, S.-P. Cai ${ }^{2}$, \\ X.-M. Zhou ${ }^{3}$, X.-M. Chen ${ }^{3}$ and X.-Y. Liu ${ }^{2}$ \\ ${ }^{1}$ The State Key Laboratory of Biotherapy, Sichuan University, Chengdu, \\ Sichuan, China \\ ${ }^{2}$ Shenzhen Key Laboratory of Ophthalmology, Shenzhen Eye Hospital, \\ Jinan University, Shenzhen, China \\ ${ }^{3}$ Regenerative Medicine Research Center, West China Hospital, \\ Sichuan University, Chengdu, Sichuan, China \\ ${ }^{4}$ Department of Ophthalmology, The Second Affiliated Hospital, \\ University of South China, Hengyang, China \\ *These authors contributed equally to this study. \\ Corresponding author: X.-Y. Liu \\ E-mail: xliu1213@126.com
}

Genet. Mol. Res. 14 (2): 4125-4132 (2015)

Received February 17, 2014

Accepted October 30, 2014

Published April 27, 2015

DOI http://dx.doi.org/10.4238/2015.April.27.27

ABSTRACT. The purpose of this study was to identify the clinical
features and mutations in the fibrillin-1 gene (FBN1) in a large Chinese
family with autosomal dominant Marfan syndrome (MFS). Seventeen
members from a Chinese family of 4 generations were included in
the study. All members underwent complete ophthalmic examination.
Molecular genetic analysis was performed on all subjects. All exons of
FBN1 were amplified by polymerase chain reaction, sequenced, and
the sequences were compared with a reference database. Variations
were evaluated in family members as well as 100 normal controls.
Changes in structure and function of the protein induced by amino
acid variation were predicted by bioinformatic analysis. Ectopia lentis, 
dolichostenomelia, arachnodactyly, and tall stature were present in all patients diagnosed with MFS. The novel heterozygous missense mutation c.2243 $\mathrm{T}>\mathrm{G}$ (p.C781W) in exon 19 of $F B N 1$ was identified in all 5 patients, but not in other family members or 100 normal controls. This mutation caused an amino acid substitution of cysteine to tryptophan at position 781 (p.C781W) of the FBN1 protein. This mutation occurred in a highly conserved region and may cause structural and functional changes in the protein according to our bioinformatic analysis. Our results suggest that the novel mutation C781W of FBN1 is responsible for the pathogenesis of MFS in this pedigree.

Key words: Ectopia lentis; Exon; FBN1; Marfan syndrome; Mutation

\section{INTRODUCTION}

Marfan syndrome (MFS) is a multisystemic, autosomal dominant disorder with pleiotropic manifestation mainly involving cardiovascular, ocular, and skeletal tissues (Milewicz et al., 1992). The estimated prevalence of MFS is 1 in 10,000-20,000 individuals (Dong et al., 2012), without gender or ethnic predisposition (Mátyás et al., 2002). The cardiovascular complications of the disorder include aortic root dilatation and dissection, mitral valve regurgitation, and mitral valve prolapse. These complications can lead to premature death if they are untreated (Murdoch et al., 1972). Skeletal abnormalities include scoliosis, jointlaxity, arachnodactyly, anterior chest deformities, and tall stature (Pyeritz and McKusick, 1979). Ocular features include myopia, retinal detachment, and lens dislocation. The latter, also known as ectopia lentis, was observed in around $80 \%$ of MFS patients (Micheal et al., 2012). Ectopia lentis is characterized by the displacement or malposition of a lens from its normal location, causing the zonular filaments to be stretched or discontinued (Liang et al., 2011).

Genetic factors are known to play important roles in the pathogenesis of MFS. Two genes, including fibrillin-1 (FBN1) and transforming growth factor beta receptor II were found to be MFS-associated genes (Zhao et al., 2013). FBN1 consists of 65 exons, spanning $235 \mathrm{~kb}$ of genomic DNA on chromosome 15q21 and encodes a secreted 350-kDa extracellular matrix glycoprotein that serves as a structural component of 10-12-nm calcium-binding microfibrils (Ramirez and Sakai, 2010). These microfibrils provide force-bearing structural support in elastic and non-elastic connective tissue throughout the body (Hubmacher et al., 2006). FBN1 is mainly composed of 3 types of repeated modules, including a cysteine-rich epidermal growth factor (EGF)-like domain, transforming growth factor beta-1 binding protein-like module, and a hybrid module (Meng et al., 2011).

There are 47 such EGF-like domain modules in FBN1, and 43 contain a calciumbinding (cb) consensus sequence and are thus referred to as cbEGF-like modules (Handford et al., 1991). To date, more than 1200 mutations have been detected in FBN1, and most affect one of the cbEGF domains, often involving 1 of the 6 highly conserved cysteine residues within the cbEGF domains (Micheal et al., 2012).

The age-related nature of some clinical manifestations as well as variable phenotypic expression of MFS may prevent accurate diagnosis, particularly in children (Loeys et al., 2001). As such, in situations of clinical uncertainty, molecular analysis of FBN1 appears to be a logical method for the clinical diagnosis of MFS (Robinson et al., 2006). To understand the 
genetic background of a 4-generation Chinese family with MFS, the primary candidate gene FBN1 was analyzed in this study.

\section{MATERIAL AND METHODS}

\section{Family recruitment and clinical examination}

A 4-generation pedigree (Figure 1) with ectopia lentis was recruited from Shenzhen Eye Hospital (Shenzhen, Guangdong, China). Consanguineous marriage was noted in the family (Figure 1). The study was approved by the Medical Ethics Committee of the Shenzhen Eye Hospital. Informed consent was obtained from all participants according to the principles of the Declaration of Helsinki. All family members included in the study underwent examinations, including slit-lamp biomicroscopy, gonioscopy, and fundus examination. All individuals in the control group were healthy and had no history of other familial inherited diseases.

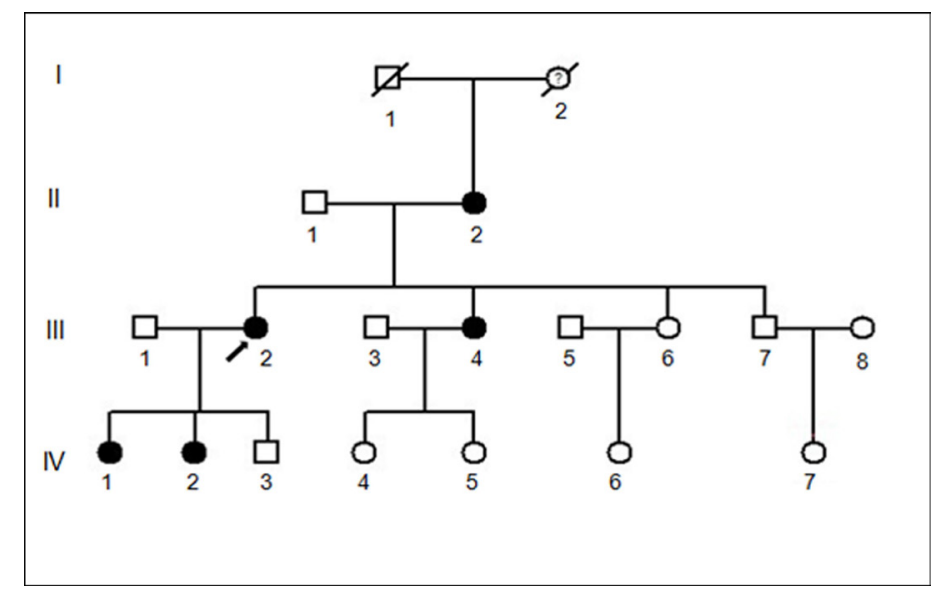

Figure 1. Pedigree of the Chinese family with MFS. Squares and circles indicate males and females, respectively, and the filled symbols represent the affected members. Slashed symbols denote deceased subjects. Symbol with a question mark in the center indicates that the diagnosis was unclear. Arrow indicates the proband. The parents of proband (II: 1 and II: 2) were consanguineous.

\section{Molecular genetic analysis}

Genomic DNA was extracted from $200 \mu \mathrm{L}$ venous blood using a Qiamp Blood Kit (Qiagen, Hilden, Germany). All procedures were performed according to manufacturer instructions. DNA integrity was evaluated by $1 \%$ agarose gel electrophoresis. All 65 exons of the FBN1 gene were amplified by polymerase chain reaction (PCR) using previously described primers (Liang et al., 2011). PCR amplification was conducted in a MyCycler thermocycler (Bio-Rad, Hercules, CA, USA). The 30- $\mu$ L PCR mixtures included 30-40 ng genomic DNA, $1.0 \mu \mathrm{M}$ of each forward and reverse primers, and $15 \mu \mathrm{L} 2 \mathrm{X}$ Taq Master Mix (including $1 \mathrm{X}$ PCR buffer, $2.5 \mathrm{mM} \mathrm{MgCl} 2,0.3 \mathrm{mM}$ of each dNTP, and 1.5 U Pfu DNA polymerase). All reagents used in this procedure were purchased from SinoBio Biltech Co., Ltd. (Shanghai, China). Sequence data were compared pairwise with the published FBN1 sequences. Muta- 
tions were named according to the nomenclature recommended by the Human Genomic Variation Society.

\section{RESULTS}

\section{Clinical findings of the pedigree}

Five of the 17 members in this 4-generation family were diagnosed as having MFS. All patients showed similar clinical manifestations, including bilateral lens dislocation (Table 1 and Figure 2). Skeletal system abnormalities such as tall stature, thin body habitus, long limbs, and arachnodactyly were observed in all patients (Figure 3). However, no patients displayed cardiovascular system abnormalities. Individuals I: 1 and I: 2 died many years ago; according to the memories of their family members, I: 2 had poor visual acuity, but no related medical records were available.

Table 1. Clinical details of the patients in the family.

\begin{tabular}{|c|c|c|c|c|c|}
\hline Patients & II: 2 & III: 2 & III: 4 & IV: 1 & IV: 2 \\
\hline Age (years) & 65 & 36 & 34 & 16 & 11 \\
\hline Gender & Female & Female & Female & Female & Female \\
\hline Ectopia lentis & + & + & + & + & + \\
\hline Myopia & - & - & - & - & - \\
\hline Strabismus & + & + & + & + & + \\
\hline \multicolumn{6}{|l|}{ Glaucoma } \\
\hline Retina detachment & - & - & - & - & - \\
\hline Height $(\mathrm{cm})$ & 168 & 174 & 171 & 165 & 162 \\
\hline $\operatorname{Arm}$ span $(\mathrm{cm})$ & 169 & 175 & 172 & 167 & 165 \\
\hline Thin body & + & + & + & + & + \\
\hline Arachnodactyly & + & + & + & + & + \\
\hline Cardiovascular system & - & - & - & - & - \\
\hline
\end{tabular}

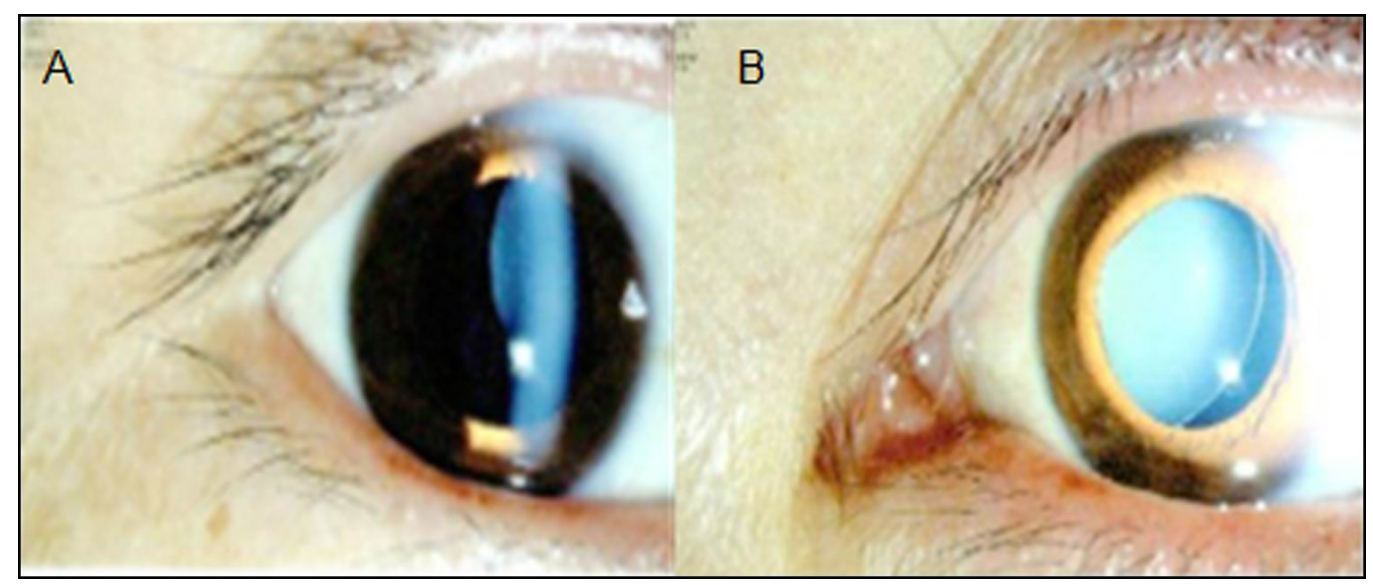

Figure 2. Anterior segment of proband (III: 2). A and B show ectopia lentis in both eyes. The pupils are dilated. The lens is dislocated nasally in the right eye (A); in the left eye (B), the lens is dislocated superiorly. 




Figure 3. Arachnodactyly of the patient IV: 2.

\section{Mutation identification and bioinformatic analysis}

Sequence analysis of $F B N 1$ revealed a heterozygous missense mutation, c.2243 $\mathrm{T}>\mathrm{G}(\mathrm{p} . \mathrm{C} 781 \mathrm{~W}$ ), in exon 19 of all 5 patients (Figure 4). No mutation was identified in other asymptomatic members of the family or 100 normal control subjects included in the study.

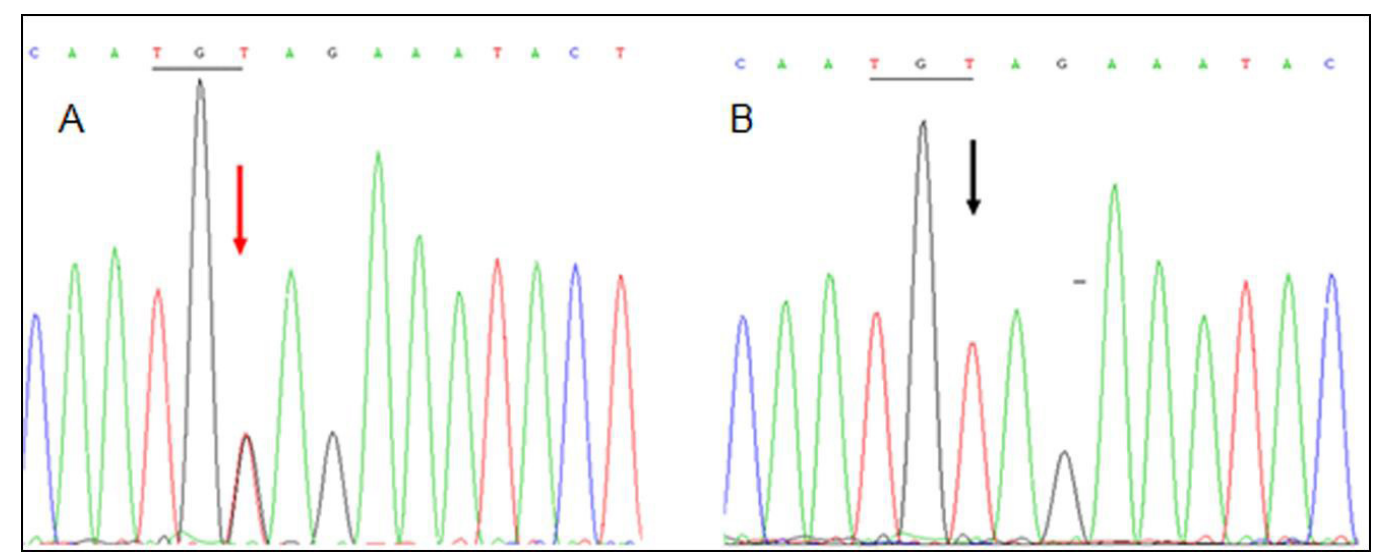

Figure 4. Sequencing results of the $F B N 1$ gene. A. A heterozygous $\mathrm{T}$ to $\mathrm{G}$ cysteine substitution at codon 781 in one patient from the family (red arrow). B. Wild-type sequence from an unaffected member.

The mutation c.2243 $\mathrm{T}>\mathrm{G}$ in exon 19 of FBN1 resulted in replacement of cysteine by tryptophan. Cysteine at position 781 was highly conserved in FBN1 based on the analysis of orthologs from 8 different species according to the online ClustalW tool (Figure 5). The p.C781W mutation of FBN1 was predicted to be "likely damaging" by polymorphism phenotyping with a score of 0.99 and "damaging" by sorting intolerant from tolerant analysis with a score of 0.00 , indicating that protein function was affected by amino acid changes (a score $<0.05$ indicates that the amino acid change affects the protein function). Secondary structure prediction using the GORIV method suggested that the p.C781 W mutation resides in the 12 th cbEGF domain, affecting 1 of the most highly conserved cysteine residues. 


\begin{tabular}{|c|c|}
\hline [Homo sapiens] & TYKCICNSGYEVDSTGKNCVDINECVLNSLLCDNG6 \\
\hline [Gallus gallus] & TYKCICNPGYEVDSTGKNCIDIDECVLNTLLCDNG \\
\hline [Mus musculus] & TYKCICNSGYEVDITGKNCVDINECVLNSLLCDNG \\
\hline [Rattus norvegicus] & TYKCICNSGYEVDITGKNCVDINECVLNSLLCDNG \\
\hline [Callithrix jacchus] & TYKCICNSGYEVDSTGKNCVDINECVLNSLLCDNGQCRNTPGSFVCT \\
\hline [Bos taurus] & TYKCICNSGYEVDSTGKNCVDINECVLNSLLCDNGQ CRNTPGSFVCT \\
\hline [Pan troglodytes] & TYKCICNSGYEVDSTGKNCVDINECVLNSLLCDNGQ CRNTPGSFVCT \\
\hline [Macaca mulatta] & TYKCICNSGYEVDSTGKNCVDINECVLNSLLCDNGQCRNTPGSFVCT \\
\hline
\end{tabular}

Figure 5. Mutation involved a highly conserved residue. The cysteine at position 781 is highly conserved for $F B N 1$, which was demonstrated by analyzing orthologs from 8 different species.

\section{DISCUSSION}

FBN1 was the first identified gene associated with the pathogenesis of MFS (Dietz et al., 1991). FBN1 mutations are thought to exert a dominant negative effect (Erentug et al., 2006). To date, more than 1000 of the mutations in this gene have been described, and these mutations are spread throughout the entire gene without a clear predilection for any given region (Boileau et al., 2005). Most of the mutations are missense mutations, and most of these are cysteine substitutions.

The coding sequence of $F B N 1$ is spread over 65 coding exons. Previous studies revealed that mutations in FBN1 frequently occur in exons 2, 15, 22, 27, 46, 55, and 62 (Faivre et al., 2008) rather than exon 19 (Micheal et al., 2012). To date, only 5 mutations have been identified in exon 19, including a frameshift mutation (2412delAT) observed in one Italian patient with clinical symptoms mainly involving the skeletal and cardiovascular systems (Pepe et al., 2001), 3 missense mutations in 3 sporadic patients (p.C781R, p.C781R, p.C776Y) in patients from Belgium with classical MFS and involvement of the cardiovascular system (Loeys et al., 2001), and a heterozygous missense mutation (c.2368T >A p.C790S) in all affected members presenting ectopia lentis, myopia, and glaucoma, but lacking the cardinal cardiovascular features of MFS in a large Pakistani family (Micheal et al., 2012). In our study, the novel heterozygous missense mutation c.2243 T>G (p.C781W) in exon 19 of FBN1 was identified in all patients in the pedigree. All patients presented bilateral lens dislocation, strabismus, tall stature, thin body habitus, long limbs, and arachnodactyly, but without cardiovascular system abnormalities. In early reports, 4 mutations identified in exon 19 were found to be associated with cardiovascular system involvement; however, this was not the case in the family described in our study.

EGF-like domains of FBN1 play a principal role in the pathogenesis of fibrillinopathies, as most of the mutations in these domains are associated with classic MFS (Schrijver et al., 1999). Among these mutations, most are located in cbEGF-like domains. Each cbEGF-like domain of fibrillin-1 contains 6 highly conserved cysteine residues that form 3 intra-domain disulfide bonds (C1-C3, C2-C4, C5-C6) and a consensus sequence for calcium binding in the NH2-terminal region (Reinhardt, 1997). Because 3 disulfide bonds are required to maintain cbEGF-like module fold, the loss or addition of cysteine residues can result in module mis- 
folding (Downing et al., 1996). Higher frequency substitutions of cysteine were observed in MFS patients with ectopia lentis (Rommel et al., 2005). This result agrees with the clinical findings in the family described herein. In our study, we identified a mutation in cbEGF modules. A missense mutation in exon 19 of FBN1 located in the cbEGF-like \#12 domain results in replacement of cysteine by tryptophan (p.C781W). Cysteine deficiency may alter the intramolecular disulfide bond arrangement, changing the secondary structure of the protein (Dietz et al., 1993). The presence of these particular clinical features such as bilateral lens dislocation, strabismus, tall stature, thin body habitus, long limbs, and arachnodactyly in all affected individuals may be due to the novel mutation p.Cys781Trp or may be associated with additional genetic factors contributing to the disease phenotype in this family.

In summary, we identified the C781W mutation in MFS patients. This study added a novel mutation to the existing spectrum of FBN1 mutations, suggesting that mutations in FBN1 are correlated with MFS as observed in this family. This study adds support for the need for genetic diagnosis of inherited disorders.

\section{ACKNOWLEDGMENTS}

The authors are deeply grateful to all of the family members for their cooperation in this study. Research supported by grants from the National Nature Science Foundation of China (\#NNSF30872830 and \#81170851).

\section{REFERENCES}

Boileau C, Jondeau G, Mizuguchi T and Matsumoto N (2005). Molecular genetics of Marfan syndrome. Curr. Opin. Cardiol. 20: 194-200.

Dietz HC, Cutting GR, Pyeritz RE, Maslen CL, et al. (1991). Marfan syndrome caused by a recurrent de novo missense mutation in the fibrillin gene. Nature 352: 337-339.

Dietz HC, McIntosh I, Sakai LY, Corson GM, et al. (1993). Level and EGF-like domain calcium binding in the pathogenesis of Marfan syndrome. Genomics 17: 468-475.

Dong J, Bu J, Du W, Li Y, et al. (2012). A new novel mutation in FBN1 causes autosomal dominant Marfan syndrome in a Chinese family. Mol. Vis. 18: 81-86.

Downing AK, Knott V, Werner JM, Cardy CM, et al. (1996). Solution structure of a pair of calcium-binding epidermal growth factor-like domains: implications for the Marfan syndrome and other genetic disorders. Cell 85: 597-605.

Erentug V, Polat A, Bozbuga NU, Polat E, et al. (2006). Cardiovascular reoperations in Marfan syndrome. J. Card. Surg. 21: 455-457.

Faivre L, Collod-Beroud G, Child A, Callewaert B, et al. (2008). Contribution of molecular analyses in diagnosing Marfan syndrome and type I fibrillinopathies: an international study of 1009 probands. J. Med. Genet. 45: 384-390.

Handford PA, Mayhew M, Baron M, Winship PR, et al. (1991). Key residues involved in calcium-binding motifs in EGFlike domains. Nature 351: 164-167.

Hubmacher D, Tiedemann K and Reinhardt DP (2006). Fibrillins: from biogenesis of microfibrils to signaling functions. Curr. Top. Dev. Biol. 75: 93-123.

Liang C, Fan W, Wu S and Liu Y (2011). Identification of a novel FBN1 mutation in a Chinese family with isolated ectopia lentis. Mol. Vis. 17: 3481-3485.

Loeys B, Nuytinck L, Delvaux I, De Bie S, et al. (2001). Genotype and phenotype analysis of 171 patients referred for molecular study of the fibrillin-1 gene FBN1 because of suspected Marfan syndrome. Arch. Intern. Med. 161: 24472454.

Mátyás G, De Paepe A, Halliday D, Boileau C, et al. (2002). Evaluation and application of denaturing HPLC for mutation detection in Marfan syndrome: identification of 20 novel mutations and two novel polymorphisms in the FBN1 gene. Hum. Mutat. 19: 443-456.

Meng B, Li H, Yang T, Huang S, et al. (2011). Identification of a novel FBN1 gene mutation in a Chinese family with Marfan syndrome. Mol. Vis. 17: 2421-2427. 
Micheal S, Khan MI, Akhtar F, Weiss MM, et al. (2012). Identification of a novel FBN1 gene mutation in a large Pakistani family with Marfan syndrome. Mol. Vis. 18: 1918-1926.

Milewicz DM, Pyeritz RE, Crawford ES and Byers PH (1992). Marfan syndrome: defective synthesis, secretion, and extracellular matrix formation of fibrillin by cultured dermal fibroblasts. J. Clin. Invest. 89: 79-86.

Murdoch JL, Walker BA, Halpern BL, Kuzma JW, et al. (1972). Life expectancy and causes of death in the Marfan syndrome. N. Engl. J. Med. 286: 804-808.

Pepe G, Giusti B, Evangelisti L, Porciani MC, et al. (2001). Fibrillin-1 (FBN1) gene frameshift mutations in Marfan patients: genotype-phenotype correlation. Clin. Genet. 59: 444-450.

Pyeritz RE and McKusick VA (1979). The Marfan syndrome: diagnosis and management. N. Engl. J. Med. 300: $772-777$.

Reinhardt DP, Ono RN and Sakai LY (1997). Calcium stabilizes fibrillin-1 against proteolytic degradation. J. Biol. Chem. 272: 1231-1236. Ramirez F and Sakai LY (2010). Biogenesis and function of fibrillin assemblies. Cell Tissue Res. 339: 71-82.

Robinson PN, Arteaga-Solis E, Baldock C, Collod-Béroud G, et al. (2006). The molecular genetics of Marfan syndrome and related disorders. J. Med. Genet. 43: 769-787.

Rommel K, Karck M, Haverich A, von Kodolitsch Y, et al. (2005). Identification of 29 novel and nine recurrent fibrillin-1 (FBN1) mutations and genotype-phenotype correlations in 76 patients with Marfan syndrome. Hum. Mutat. 26: 529-539.

Schrijver I, Liu W, Brenn T, Furthmayr H, et al. (1999). Cysteine substitutions in epidermal growth factor-like domains of fibrillin-1: distinct effects on biochemical and clinical phenotypes. Am. J. Hum. Genet. 65: 1007-1020.

Zhao F, Pan X, Zhao K and Zhao C (2013). Two novel mutations of fibrillin-1 gene correlate with different phenotypes of Marfan syndrome in Chinese families. Mol. Vis. 19: 751-758. 